\title{
Non-linear Ultrasonic Spectroscopy as an Assessment Tool for the Structure Integrity of Concrete Specimens
}

\author{
M. Korenska* \& M. Matysik \\ Department of Physics, Faculty of Civil Engineering, Brno University of Technology, Brno, Czech Republic \\ *Corresponding author: korenska.m@fce.vutbr.cz
}

DOI: $10.2478 / v 10158-010-0003-3$

\begin{abstract}
On the basis of non-linear effect studies, new diagnostic and defectoscopic methods have been designed which are based on the elastic wave non-linear spectroscopy. The non-linear ultrasonic spectroscopy brings new prospects into the acoustic non-destructive testing of material degradation. Poor material homogeneity and, in some cases, the shape complexity of some units used in the building industry, are heavily restricting the applicability of "classical" ultrasonic methods. These linear acoustic methods focus on the energy of waves, which are reflected by structural defects, variations of the wave propagation velocity, or changes in the wave amplitude. However, none of these "linear" wave characteristics is as sensitive to the structure defects as the specimen non-linear response. In this way, non-linear methods thus open new horizons in non-destructive ultrasonic testing, providing undreamed-of sensitivities, application speeds, and easy interpretation. One of the fields in which a wide application range of non-linear acoustic spectroscopy methods can be expected is civil engineering. It is predicted that these advanced techniques can contribute a great deal to the improvement and refinement of the defectoscopic and testing methods in the building industry. The present paper deals with analyzing one of the non-linear ultrasonic defectoscopic methods from the viewpoint of its application to the concrete specimen structural integrity evaluation. Both intact specimens and specimens subject to various kinds of stress have been tested. The effect of structural defects on the elastic wave propagation has been studied.
\end{abstract}

KEY WORDS: Non destructive testing, nonlinear ultrasonic spectroscopy, concrete, structural integrity.

\section{INTRODUCTION}

Thanks to the turbulent development of concrete and reinforced concrete buildings taking place in the last century, the condition of concrete and reinforced concrete became a hot topic in the last decade. Concrete has proved to be a durable construction material in the past, however, concrete structures often experienced degradation after years of service. Rehabilitation techniques have been developed in foreign countries over several decades, showing a rapid development in general. However, the absence of an acceptable, relatively fast and cheap monitoring method, which would be capable of detecting structure faults at an early stage, and thus making a simple and cost-effective maintenance possible, is still persisting (Macecek, 2003). 
On the basis of non-linear effect studies, new diagnostic and defectoscopic methods have been designed which are based on the elastic wave non-linear spectroscopy (Abeele and al. 2000, Johnson, 1999). Recently, various papers have been published on the theoretical or experimental verification of different methods in some application areas. One may state that it is still in its infancy, regarding the topic complexity, the requirements for newly developed special instrumentation and a high potential application diversity, the research and development of the respective methods, the required instrumentation and, last but not least, the practical applications of these methods,. On the other hand, most published papers, as well as our experience, show these methods to be highly promising for both defectoscopy and material testing purposes in the near future.

Our experimental and theoretical research work on Non-linear ultrasonic spectroscopy is focused on investigating non-linear ultrasonic transmission through building components from different inhomogeneous materials, such as ceramic, concrete, and reinforced concrete (Korenska \& Manychova, 2008).

\section{EXPERIMENTAL ARRANGEMENT}

The method with one exciting harmonic ultrasonic signal was applied, where the generation of higher harmonics by a monochromatic excitation is evaluated. The experimental set-up and testing of its component units have been described in detail previously (Manychova, 2007) and will be only briefly described here, Fig. 1.

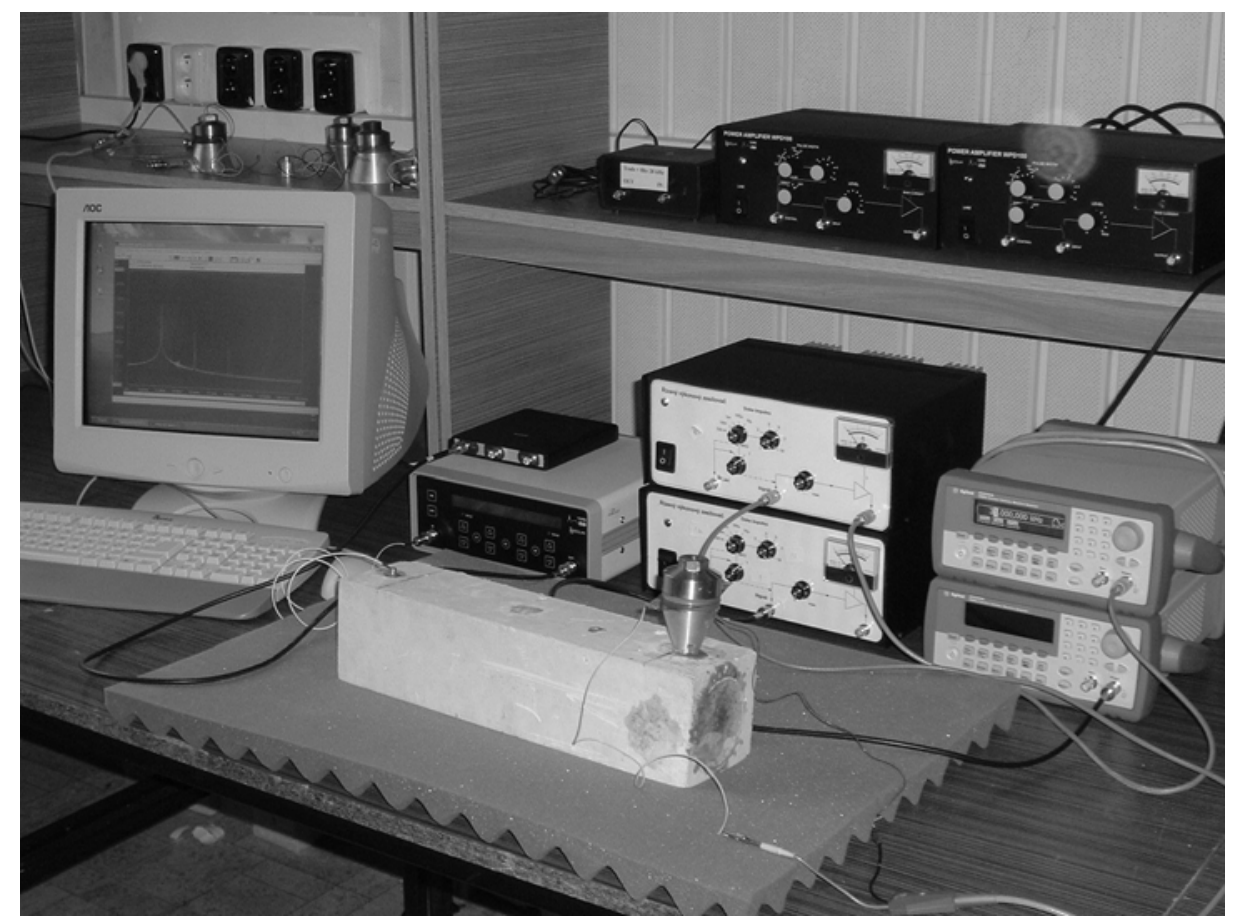

Figure 1: Experimental arrangement, photo of the non-linear ultrasonic equipment with a test specimen

The measuring apparatus consists of two principal parts, namely, a transmitting section and a receiving and measuring section. The transmitting section consists of four functional 
blocks: a controlled-output-level harmonic signal generator, a low-distortion $100 \mathrm{~W}$ power amplifier, an output low-pass filter to suppress higher harmonic components and ensure the high purity of the exciting harmonic signal. The main chain of the receiving section includes an input amplifier with filters designed to minimize the receiving chain distortion and a band-pass filter amplifier. Having been amplified, the pickup output signal was then fed into a THPS3-25 HandyScope3 measuring instrument to be sampled and analyzed.

To interpret the recorded data properly, each of the measuring instruments must answer the demands of high linearity. For the purpose of improving the reliability and accuracy of the non-linear experiments and of minimizing the error effects the attention was focused on the transmission between transmitter and sensors. Elements meeting the given requirements were chosen (Korenska at al. 2006). A program package to control the measuring process, the data processing, and the evaluation makes for an indispensable tool. The measurement results were represented in the form of frequency spectra.

\subsection{Intact Concrete Specimens}

In the first stage of the experiment, we tried to find out whether the high inhomogeneity of the concrete material gives rise or does not give rise to the non-linear phenomena. Two groups of specimen were denoted $\mathrm{A}$ and $\mathrm{B}$, both featuring the same concrete mix charge. Each group comprised of 10 specimens of dimensions $150 \mathrm{~mm} \times 150 \mathrm{~mm} \times 150 \mathrm{~mm}$, the A group specimen having been aerated, whereas the B group ones were non-aerated.

\subsubsection{Measurement Results}

Fig. 2a) shows the results obtained from A-group specimens. It shows the frequency spectrum of the A3 specimen response to the harmonic ultrasonic excitation at a frequency of $29 \mathrm{kHz}$, as captured by a K33 sensor. The first five harmonic frequencies appear to be emphasized, their amplitudes decreasing with the serial number $n$. Similar results have been obtained from B-group specimens, as is seen in Fig. 2b), showing the frequency spectrum of the B4 specimen. A comparison of the diagrams shows that the aerated specimen of the Agroup suppresses the higher harmonic frequencies more intensely, which is apparently due to the air pores in the specimen structure.

All of the specimens tested showed the same amplitude decreasing trend for the harmonic frequency increasing order.

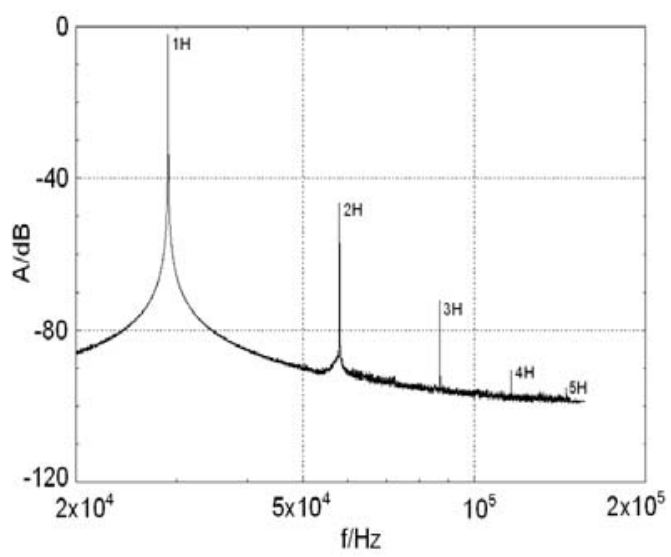

a)

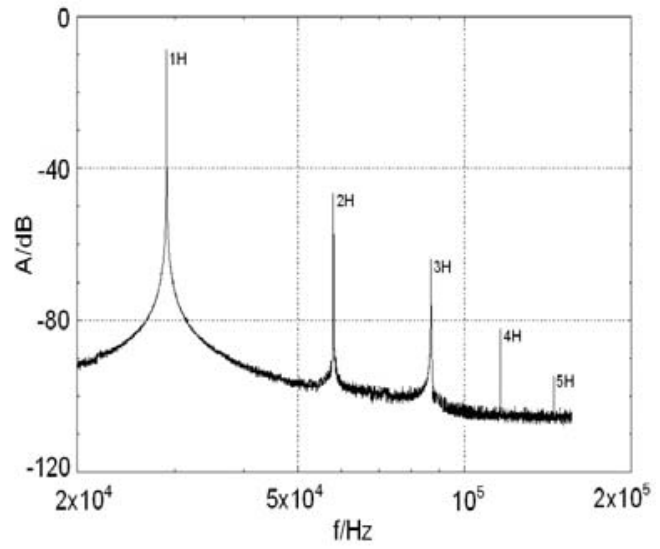

b)

Figure 2: Frequency spectrum - a) A3 specimen, b) B4 specimen. 


\subsection{Freeze - Thaw Cycle Degraded Concrete Specimens}

In the second stage, we studied the concrete specimen structure having been stressed by thermal shocks. The concrete specimens were stressed by recurrent freeze-thaw cycles. Measurements were realized after 25 and 50 freeze-thaw cycles.

\subsubsection{Measurement Results}

Figure 3 shows the high harmonics' amplitudes relative to the first harmonic's amplitude for the A2 specimen in all three conditions: pre-degradation (0 cycle), after 25 freeze-thaw cycles, and after 50 freeze-thaw cycles. The harmonic component amplitudes decrease with the serial number $n$ increase in the case of pre-degradation condition. This is evidence of the fact that this inhomogeneous material creates no source of non-linear phenomena. The transfer characteristic, which corresponds to the same specimen having been subjected to 25 freeze-thaw cycles, does show a non-linearity. It becomes evident from a distinct drop of the fourth harmonic's amplitude $(4 \mathrm{H})$, which is exceeded by the fifth harmonic's amplitude (5H). Relative amplitude values corresponding to the specimen having undergone 50 freezethaw cycles also show non-linearities. In this case they consist of a drop of the third harmonic's amplitude and an increase of the fourth harmonic $(4 \mathrm{H})$.

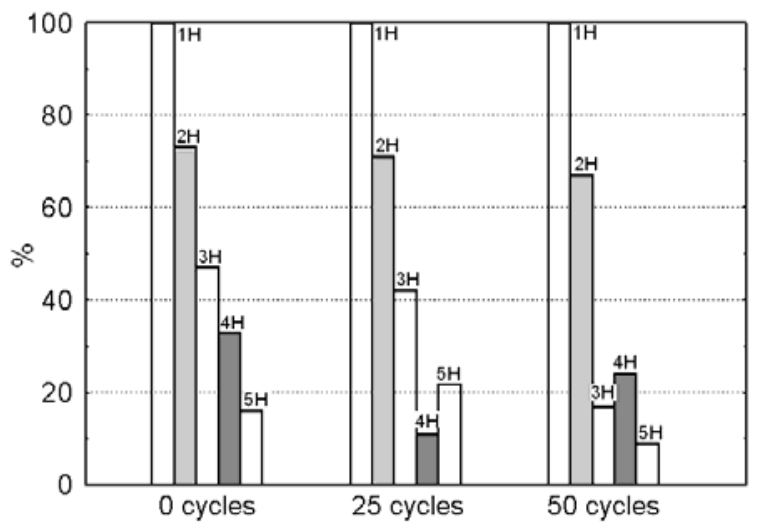

Figure 3: Relative amplitude values.

\subsection{Polymer-Fibre-Filled Concrete Specimens}

Finally, we tested concrete specimens of dimensions $100 \mathrm{~mm}$ x $100 \mathrm{~mm} \mathrm{x} 400 \mathrm{~mm}$ which had been filled with polymer fibres. The specimens were examined for flexural tensile strength in a two-weigh pressing machine. The load test was terminated as soon as visible cracks appeared. These cracks closed again after the strain was removed. In the case of the TP7 specimen a visible crack remained after the specimen had been relieved. The transmitter and sensor configuration is shown in Fig. 4.

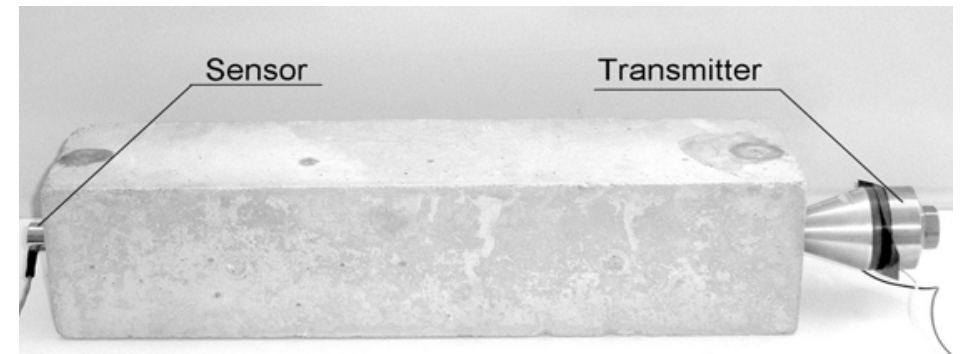

Figure 4: Location of the exciter and the sensor on the specimen under test. 


\subsubsection{Measurement Results}

Pre-test measurements of the TP7 specimen are shown in Fig. 5. The shape of the frequency spectrum corresponds to that of the intact specimen and higher harmonic amplitudes are decreasing with their serial number.

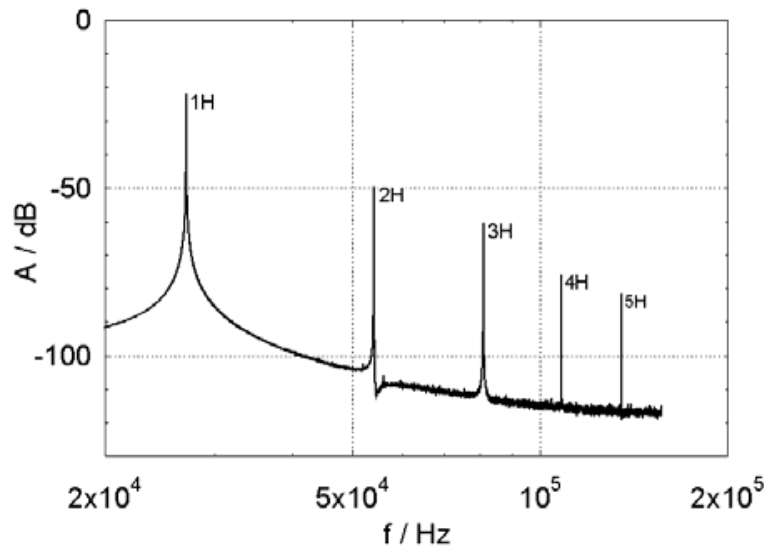

Figure 5: Pre-test frequency spectrum of TP7 specimen.

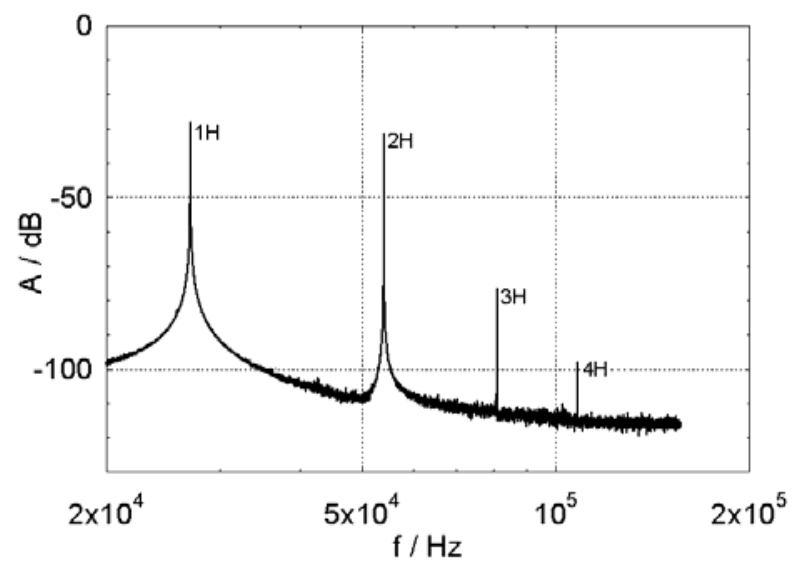

Figure 6: Frequency spectrum of TP7 after load test.

Fig. 6 shows the frequency spectrum of the same specimen after the load test was carried out in the pressing machine. When compared with Fig.5, the response function shows the higher harmonic frequencies to have lower amplitudes. The fifth harmonic has disappeared entirely which is probably due to the structure-degradation-induced higher attenuation. Another change consists of emphasizing the second harmonic's amplitude with respect to the first harmonic, as well as both the third and fourth harmonics.

\section{CONCLUSION}

This paper presents our first results of concrete specimen structure testing by means of nonlinear ultrasonic spectroscopy using a single exciting harmonic frequency.

To properly interpret the measuring results, the transfer function of the whole measuring set-up must be determined and all measuring instruments must comply with high quality standards. It is therefore essential to minimize any harmonic distortion in the signal pick-up and amplification path by eliminating any spurious signals ( $3 f_{1}$ parasitic signal, noise) from the measuring apparatus stages. It should be noted that a perfect mechanical coupling must be ensured between the exciter and the specimen and, further, between the specimen and the pick-up element during the experiment setup process. 
Our measurements show that the effect of a material inhomogeneity is very low in the case of non-linear ultrasonic spectroscopy, its non-linear effect being substantially lower than in the case of common defects.

Furthermore, it has been proved that structure defects, which are due to the freeze-thaw cycles as well as mechanical strain, have caused changes in the signal propagation frequency dependence function. Structural integrity deterioration has given rise to non-linear effects in the transfer functions, which correlated with the deterioration degree.

Verification measurements of fundamental physical quantities have also proved the structural degradation to be caused by specimen mechanical loading.

\section{Acknowledgments}

This research is supported by GA CR 103/09/1499 and MSM 0021630519 projects.

\section{REFERENCES}

Van Den Abeele, Johnson, P. A. and Sutin, A., 2000. Nonlinear Elastic Wave Spectroscopy (NEWS) techniques to discern material damage. Part I: Nonlinear Wave Modulation Spectroscopy (NWMS), Research on NonDestructive Evaluation 12, pp. 17-30.

Van Den Abeele, Carmeliet, J., James, A. Ten Cate, Johnson, P. A., 2000. Nonlinear Elastic Wave Spectroscopy (NEWS) Techniques to Discern Material Damage. Part II: Single Mode Nonlinear Resonance Acoustic Spectroscopy. Research on NonDestructive Evaluation 12, 31- 42.

Johnson, P. A., 1999. The new wave in acoustic testing. The J. Inst. Materials 7, 544-546.

Hajek, K., Sikula, J., 2005. Testing of Low-Current Contacts Quality and Reliability by Using Third Harmonic Distortion. IEEE Trans. on Components and Packaging Technologies 28, pp.717 - 720 .

Korenska, M, Manychova, M., 2008. Nonlinear Ultrasonic Spectroscopy Used to Detection of Ceramic Structure Damage. Nonlinear Acoustics- Fundamentals and Applications, ISNA18, pp. 541-544, Ed. Bengt O. Enflo, Claes M. Hedberg, Leif Kari, American Institute of Physics, New York 2008.

Korenska, M., Pazdera, L., Matysik, M., 2006. Testing of Pickups for Single-exciting-signal Non-linear Acoustic Spectroscopy. International Workshop Physical and Material Engineering, Slovak University of Technology in Bratislava, pp. 70-75, Ed. Palackova, A.

Manychova, M. 2007. Measuring Setup for the Nonlinear Ultrasonic Spectroscopy Method. 37th International Conference Defektoskopie 2007, pp. 147-151, Ed. Korenska, M.

Převorovský, Z., Van Den Abeele, K.E. 2004. Calibration Samples for Use in Nonlinear Ultrasonic Spectroscopy. 34th International Conference and NDT Technique Exposition Defektoskopie 2004, pp. 347-349, Ed. Mazal, P.

Macecek, M., 2003. Ultrasonic Concrete Testing. $33^{\text {rd }}$ International Conference Defektoskopie 2003, p. 117-132, Ed. Mazal, P. 